\title{
Segmentation of MR Brain Image using Multi Atlas Matching Technique
}

\author{
M.A.P. Manimekalai, N.A. Vasanthi, \\ Thusnavis Bella Mary
}

\begin{abstract}
Detection of brain tumor from Magnetic Resonance Image (MRI) image has become one of the most active researches in the field of medical image processing. Segmentation and Detection of tumor play a major role in biomedical imaging. In this research, tumor segmentation process is done with MR brain image. The proposed method contain image pre processing, image enhancement using Contrast Limited Adaptive Histogram Equalization (CLAHE) and segmentation with multi atlas matching and detection of tumor. The proposed work segment the tumor region precisely from the MR brain image. The experimental result gives an average of 0.85 Dice Similarity Co efficient (DSC), which indicates that the proposed method is efficient in segmentation and detection of the tumor region from the MR brain image.
\end{abstract}

Keywords--- Image Enhancement, Image Fusion, MR Images, Atlas Matching, PSNR.

\section{INTRODUCTION}

Traumatic brain injury (TBI) image occurs due to the external force which injures the brain [1]. The injury is caused by various factors which includes fall from workplace, road accidents, and due to blast injury, etc. Based on Glasgow Coma Scale (GCS) and Loss of Consciousness (LOC), the brain injury is categorized as mild, moderate or severe injury. In the year 2016, survey from National Health Interview anticipated that nearly one million people were affected with TBI, 200,000 people died and nearly 1 million required rehabilitation services each year in India. The detection of brain injury is an important and difficult tasks in the field of medicine.

Hence an automatic detection method for brain injury is highly desirable. For the purpose of automatic detection soft computing techniques are integrated with the biomedical imaging techniques such as Computed Tomography (CT) and Magnetic Resonance Imaging (MRI).In brain injury MR images, the blood clot possesses Isodense or Hypodense appearance immediately after a week or month of occurrence.

Soft computing techniques which were designed for diagnosis, provides better results for real world problems such as detection of brain injury, are difficult to analyze by conventional mathematical models. The popular technique for image enhancement is histogram equalization which gives good performance in natural images. However, it suffers from noise amplification. Adaptive Histogram Equalization (AHE) more suitable for enhancing the local

Revised Version Manuscript Received on August 14, 2019.

M.A.P. Manimekalai, Department of ECE, Karunya Institute of Technology and Sciences, Coimbatore. T.N, India. (e-mail: manisankar2008@gmail.com)

N.A. Vasanthi, Department of CSE, Adithya Institute of Technology, Coimbatore, T.N, India. (e-mail: vasanthi.au@gmail.com)

Thusnavis Bella Mary, Department of ECE, Karunya Institute of Technology and Sciences, Coimbatore. T.N, India (e-mail: bellamary@karunya.edu) contrast of an image [2]. To overcome this issue, CLAHE algorithm is proposed, that can reduce amplification of noise in homogeneous region of an image.

From the enhanced MR images, multi atlas based segmentation is preferred for label fusion process. In this algorithm, the poorly performing labels are discarded, as these labels provide no longer contribution for the estimation of ground truth segmentation

Image enhancement using Discrete Wavelet Transform (DST) and CLAHE approach [3],[4] are used for low contrast images. In the proposed method, the Hue component is unchanged while the Luminance component is modified using CLAHE and the Saturation components are up-scaled using derived mapping function on the approximate components of its discrete wavelet transform. This paper concludes that the contrast enhancement technique is used to enhance color images captured under poor illumination and varying environmental conditions.

Multi-atlas based segmentation [5] that let fully automatic segmentation of image populations, exhibit a large variability in shape and image quality. This paper concludes that Selective and Iterative Method for Performance Level Estimation (SIMPLE) algorithm for label fusion process provides accurate results by employing the local information present in the propagated segmentations, otherwise the labels gets discarded.

\section{METHODOLOGY}

In this research, the Traumatic Hematoma MR images are taken as input and pre processed by using Histogram Equalization (HE).

The pre-processed image has high contrast, an increased dynamic range and the Probability Density Function (PDF) of the output will be uniform.

As the contrast of the lesion region is generally poor in brain injury CT images, the CLAHE method is used for contrast enhancement by limiting the maximum slope in transformation function.

The CLAHE method doesn't increase the inherent information content of the data, rather it increases the dynamic range of the chosen features so that it can be detected easily.

The enhanced MR image is segmented by using Multi atlas based segmentation for label fusion process. In this algorithm, the poorly performing labels are discarded, because these labels provide no longer contribution for the estimation of ground truth segmentation. The flow diagram of our proposed method is shown in figure 1. 


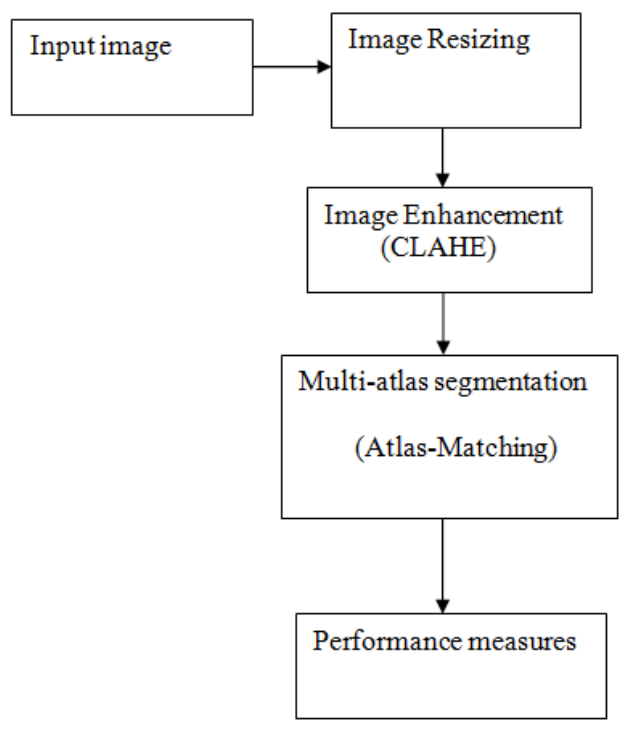

Figure 1: Flow diagram of the proposed method

\subsection{Image Enhancement}

Image Enhancement is an important image processing task. The purpose of Image Enhancement is to improve the perception of information in medical images for human viewers to get a better accuracy. It sharpens the image features such as edges, boundaries, or contrast to make a graphic display more helpful for analysis.

\subsubsection{Need for Contrast Enhancement}

Contrast Enhancement is focused on improving the contrast in an image. Contrast of an image is determined by its dynamic range, which is defined as the difference between lowest and highest intensity level. It has been used in enhancing the visual quality of low contrast images. In brain injury MRI images, the blood clot possesses Isodense or Hypodense or hypodense appearance immediately after a week or month of occurrence. Highlighting the soft tissue areas will help to detect the haematoma with better accuracy. Figure 2 shows the various lesion density regions in brain injury CT images based on the occurrence of injury.

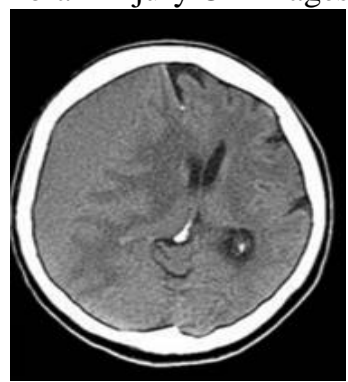

(a)

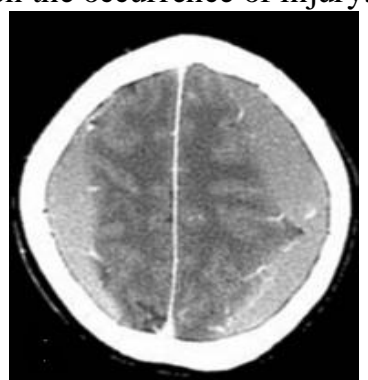

(b)

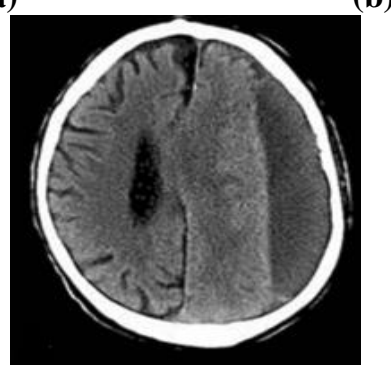

(c)

Figure 2: Various Lesion density Regions in brain Injury CT images (a) Hyperdense (b) Isodense (c) Hypodense

CLAHE transforms the image into a more appropriate appearance for the application of the medical image fusion algorithm. This method not only gives flat histogram but also enhances image contrast. CLAHE is applied on small regions in the image called tiles rather than the entire image. The block diagram of CLAHE is shown in figure 3.

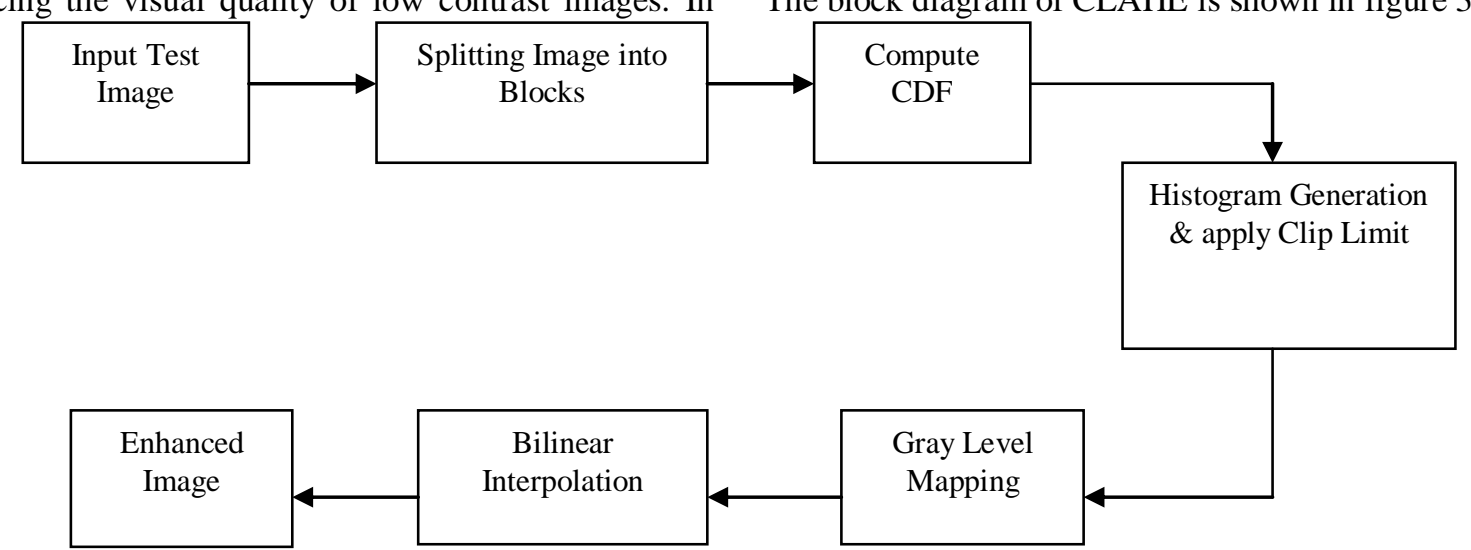

Figure 3: Block Diagram of CLAHE

Each row and each column in the image has eight tiles. The clip limit is a real scalar value in the range [0 1], that indicates a contrast enhancement limit. Larger value result in high contrast .A clip limit of 0.01 is used, which enhance the various lesion appearances in the brain injury MRI images. Higher values of the number of histogram bins used in building a contrast enhancing transformation, results in dynamic range at a cost of slower processing speed. Thus a value of 256 is used in CLAHE for every tile.

Each tile's contrast is enhanced, so that the histogram of the output region approximately matches the histogram specified by the 'Distribution' parameter. The exponential distribution parameter gives better contrast enhancement in lesion regions than uniform distribution. The Cumulative
Probability Distribution (CPD) for each pixel gets computed.

Equation 1.1 shows the formula of exponential distribution for transformed gray levels in order to calculate the new pixel value.

$$
\mathrm{g}=\left(\mathrm{g}_{\text {max }}-\mathrm{g}_{\text {min }}\right) \times \mathrm{P}(\mathrm{f})+\mathrm{g}_{\text {min }}
$$

To eliminate the artificially induced boundaries [7], the neighbouring tiles are combined using bilinear interpolation]. 
The bilinear interpolation uses only the 4 nearest pixels values, located in diagonal directions from a given pixel, to find the appropriate intensity values of the corresponding pixel.

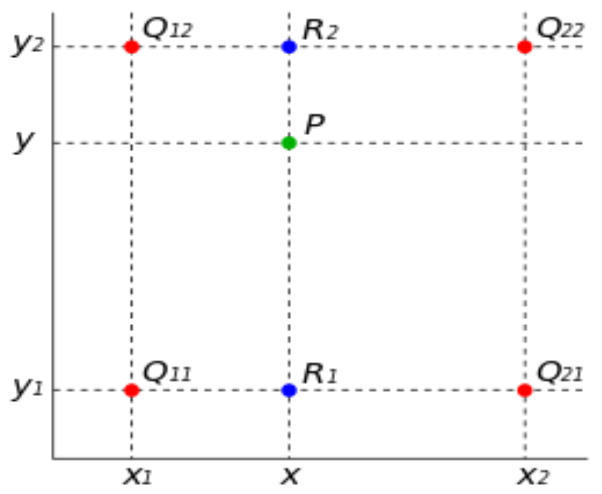

Figure 4: Example for Bilinear Interpolation

Figure 4 shows an example for bilinear interpolation in $\mathrm{X}$ and $\mathrm{Y}$ direction. For each tile block, the CPD of corner tile gets computed. The linear interpolation between previous and current pixel is carried out in one direction and it is followed in other direction of $2 \mathrm{D}$ which is found nearer to corner tiles. The new intensity at location $(\mathrm{P})$ is calculated via bilinear interpolation of CPD values at the center of 4 neighbouring tiles.

Equation 1.2 shows the formula for calculating the linear interpolation in $\mathrm{X}$-direction

$$
\begin{array}{r}
f\left(X, Y_{1}\right)=\frac{X_{2}-X}{X_{2}-X_{1}} f\left(Q_{11}\right)+\frac{X-X_{1}}{X_{2}-X_{1}} f\left(Q_{21}\right) \\
f\left(X, Y_{2}\right)=\frac{X_{2}-X}{X_{2}-X_{1}} f\left(Q_{12}\right)+\frac{X-X_{1}}{X_{2}-X_{1}} f\left(Q_{22}\right)
\end{array}
$$

Equation 1.3 shows the formula for calculating the linear interpolation in Y-direction

$$
\begin{gathered}
f(X, Y)=\frac{Y_{2}-Y}{Y_{2}-Y_{1}} f\left(X, Y_{1}\right)+\frac{Y-Y_{1}}{Y_{2}-Y_{1}} f\left(X, Y_{2}\right) \\
f(X, Y)=\frac{1}{\left(Y_{2}-Y_{1}\right)\left(X_{2}-X_{1}\right)}\left(X_{2}-X-X-X_{1}\right)+\left(\begin{array}{l}
f\left(Q_{11}\right) \\
f\left(Q_{21}\right)
\end{array}\right.
\end{gathered}
$$

where,

$$
\begin{aligned}
\mathrm{Q}_{11}=\left(\mathrm{X}_{1}, \mathrm{Y}_{1}\right) \mathrm{Q}_{12} & =\left(\mathrm{X}_{1}, \mathrm{Y}_{2}\right) \mathrm{Q}_{21}=\left(\mathrm{X}_{2}, \mathrm{Y}_{1}\right) \\
\mathrm{Q}_{22} & =\left(\mathrm{X}_{2}, \mathrm{Y}_{2}\right)
\end{aligned}
$$

The same steps are repeated for every pixel in the image. The enhancement of the contrast which is obtained especially in homogeneous areas can be limited to avoid amplifying any noise that might be present in an image. This method reduces the visual distortion of image by modifying the gray scale values according to modified histograms after combining the neighbouring tiles.

\subsection{Multi Atlas Segmentation}

Multi atlas segmentation uses multiple atlases instead of using single atlas in order to avoid the non-systematical errors in the labels. The SIMPLE algorithm is preferred for label fusion where the low performance labels get discarded in order to obtain accurate segmentation results.

In SIMPLE algorithm, the atlas selection strategy is important to obtain true segmentation of haematoma in brain injury images. For the selection of atlases, the mutual information between the target image and registered pre labelled image is calculated[8]. Equation 1.4 shows the formula of atlas selection for $\mathrm{i}$-th registered label MI $(\mathrm{Ii}, \mathrm{T})>$ median $\{\mathrm{MIj}(\mathrm{Ij}, \mathrm{Tj}), \quad$ where $\mathrm{j}=1, \ldots, 11\}$

The spatial domain fusion scheme uses the pixel average method to fuse the input image and the selected atlas images. From the fused output, the Sum of Absolute Difference (SAD) method [9][10] is used to find the measure of similarities in input image to obtain the segmentation of haematoma.

\subsection{Performance Metrics}

$$
\begin{aligned}
& \mathrm{MSE}=\frac{1}{\mathrm{~N} \times \mathrm{M}} \sum_{i=0}^{N-1} \sum_{j=0}^{M-1}[\mathrm{X}(\mathrm{i}, \mathrm{j})-\mathrm{Y}(\mathrm{i}, \mathrm{j})]^{2} \\
& \mathrm{PSNR}=10 \log _{10} \frac{255^{2}}{\mathrm{MSE}}(\mathrm{dB}) \\
& \mathrm{DSC}=2 \times \frac{\mathrm{X} \cup \mathrm{Y}}{|\mathrm{X}|+|\mathrm{Y}|}
\end{aligned}
$$

Equation 1.5, 1.6 and 1.7 shows the formula for MSE, PSNR and DSC parameters.

\section{RESULTS AND DISCUSSIONS}

\subsection{MR Input Image}

In figure 5 shows the input MR image. It has the RGB component present in it which has to be further enhanced and segmented.

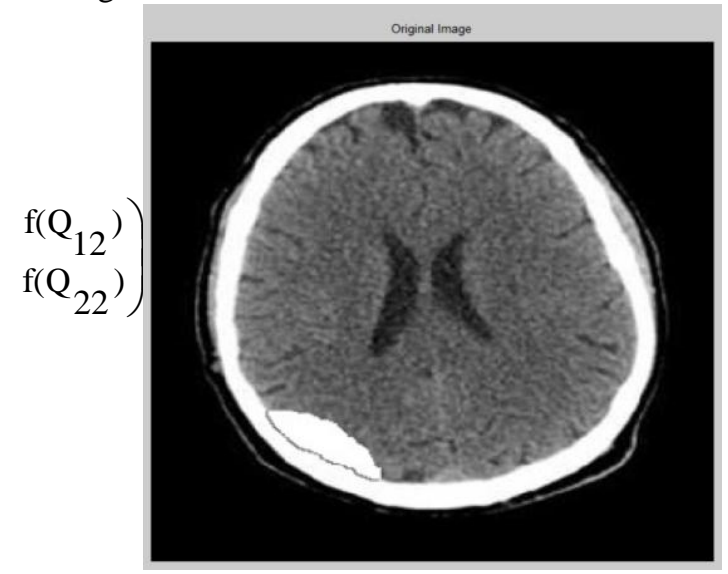

Figure 5: Input Image

\subsection{Pre-Processing}

In this stage the MR input image is further processed using the image enhancement method CLAHE. 
CLAHE transforms the image into a more appropriate appearance for the application of the medical image fusion algorithm. This method not only gives flat histogram but also enhances image contrast. CLAHE operates on small regions called tiles rather than the whole image. The enhanced image is shown in figure 6

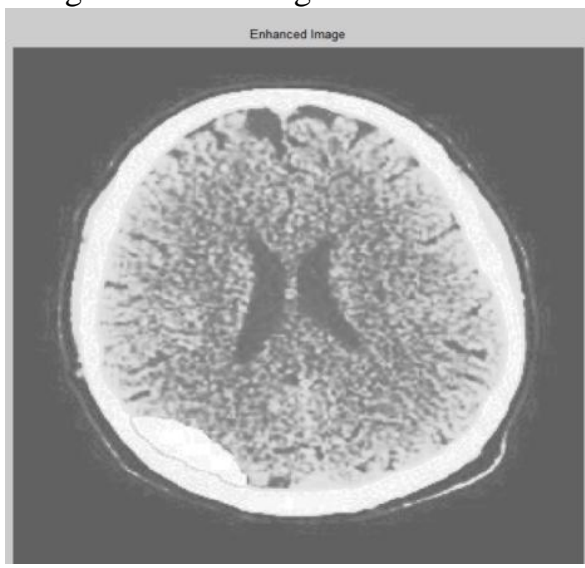

Figure 6: Enhanced Image

\subsubsection{Performance Estimation of CLAHE}

The Mean Square Error (MSE), Peak Signal to Noise Ratio (PSNR) and Dice Similarity Coefficient (DSC) are calculated in order to estimate the performance between the original and enhanced CLAHE images. Table 1 shows the performance estimation of CLAHE.

Table 1: Performance Estimation of CLAHE

\begin{tabular}{|l|l|l|l|l|}
\hline S.No & $\begin{array}{l}\text { Test } \\
\text { Image } \\
\text { Category }\end{array}$ & $\begin{array}{l}\text { Mean } \\
\text { Square } \\
\text { Error } \\
\text { (MSE) }\end{array}$ & $\begin{array}{l}\text { Peak } \\
\text { Signal to } \\
\text { Noise } \\
\text { Ratio } \\
\text { (PSNR) } \\
\text { (dB) }\end{array}$ & $\begin{array}{l}\text { Dice } \\
\text { Similarity } \\
\text { Coefficient } \\
\text { (DSC) (\%) }\end{array}$ \\
\hline 2 & $\begin{array}{l}\text { Mild } \\
\text { Category } \\
\text { (IMG001) }\end{array}$ & 13.05 & 27.61 & 99 \\
\hline 3 & $\begin{array}{l}\text { Normal } \\
\text { Category } \\
\text { (IMG010) }\end{array}$ & 5.26 & 30.74 & 77 \\
\hline $\begin{array}{l}\text { Severe } \\
\text { Category } \\
\text { (IMG007) }\end{array}$ & 10.46 & 30.42 & 73 \\
\hline
\end{tabular}

\subsubsection{Histogram Equalisation}

It is based on calculating the cumulative distributive function for the given probability density function. After the transformation, the image will have an increased dynamic range, high contrast and probability function of the output will be uniform. Figure $7 \&$ Figure 8 shows the original and enhanced histogram images respectively.

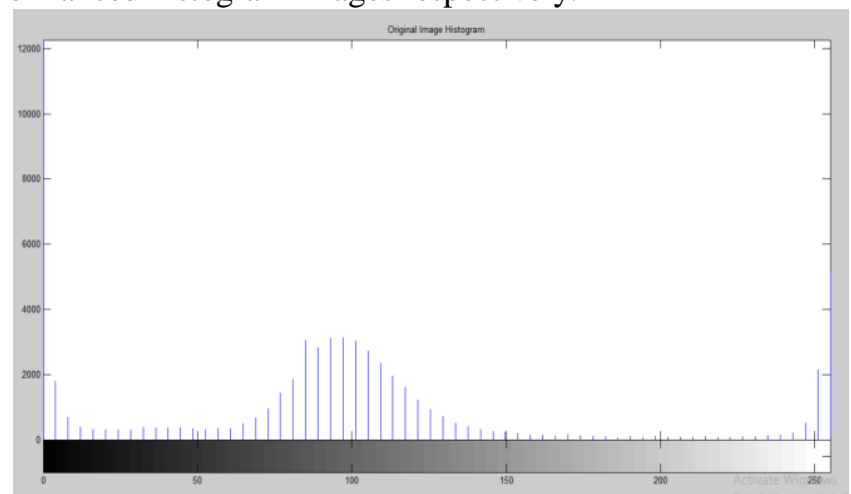

Figure 7: Original Image Histogram

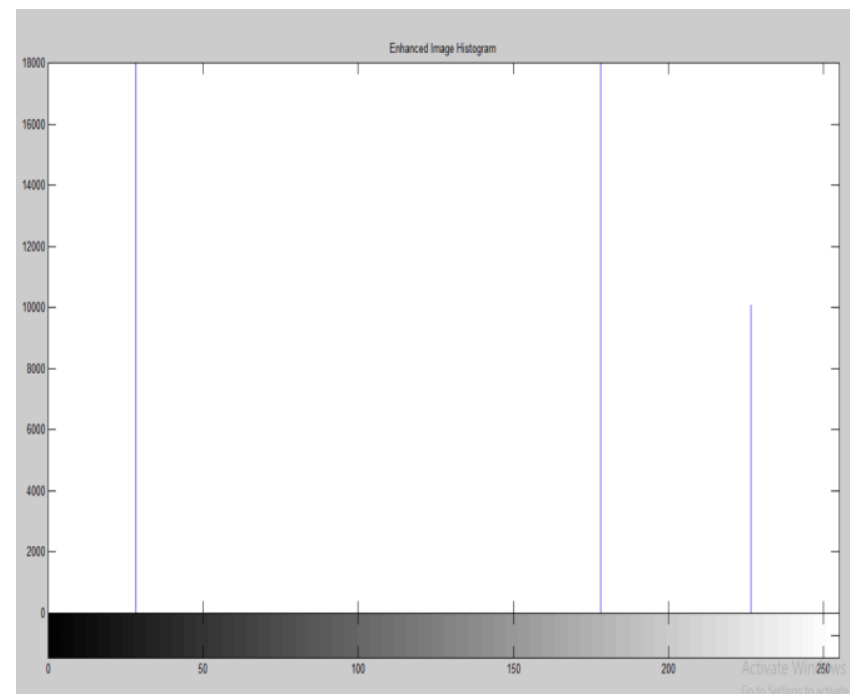

Figure 8: Enhanced Image Histogram

\subsection{Segmentation}

The goal of segmentation is to simplify and/or change the image representation.

\subsubsection{RGB Segmentation}

The RGB color model is an additive color model in which three primary colors such as red, green, and blue light are added together in different ways to reproduce a broad array of colors. The RGB color model is used for the sensing, representation, and display of images in electronic systems, such as televisions and computers, though it has been used in conventional photography. Figure 9 shows the RGB segmented image

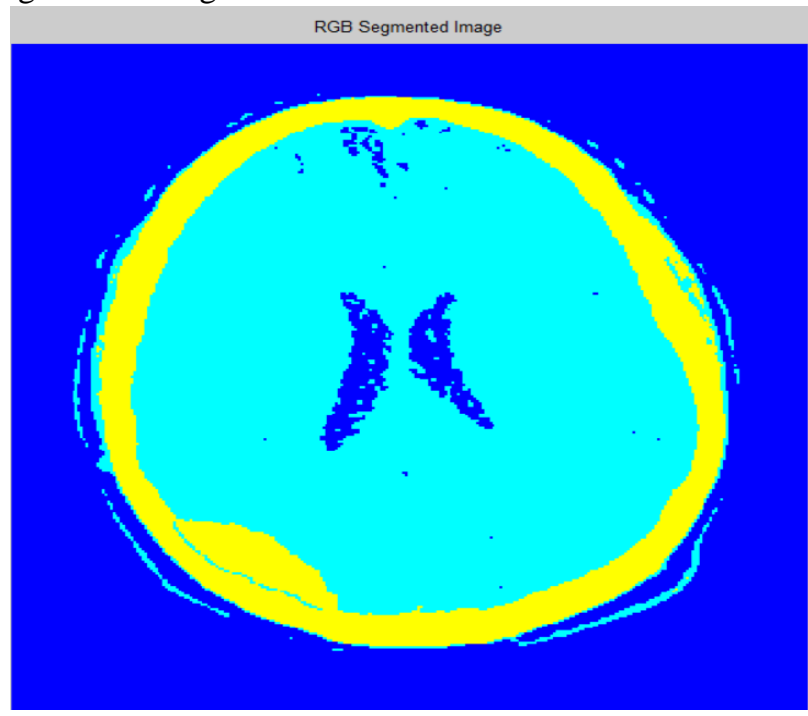

Figure 9: RGB Segmented Image

\subsubsection{Target Edge Detection}

Target edge detection is based on canny edge detection technique which is used to extract the structural information from the different vision objects and dramatically reduce the amount of data to be processed. Figure 10 shows the target edge detected image. 


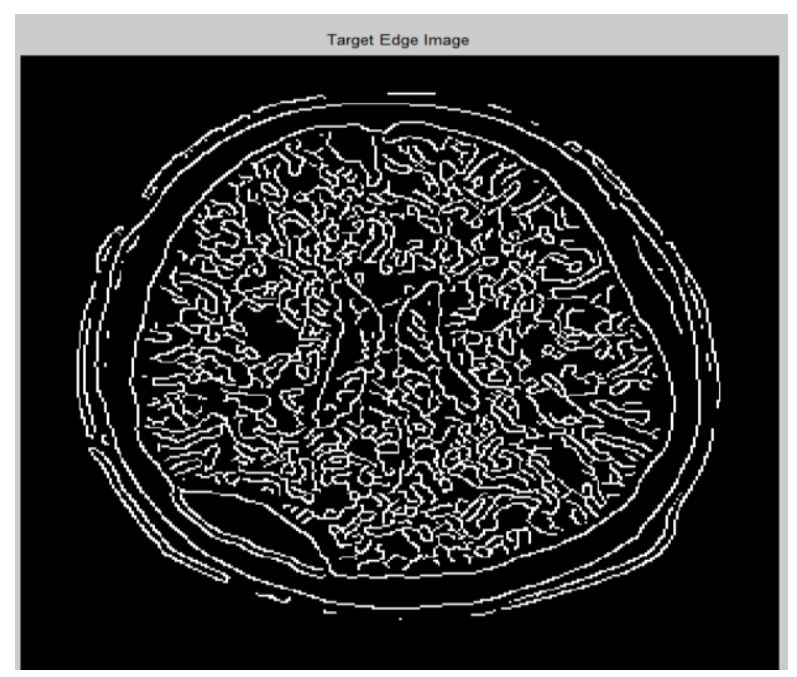

Figure 10: Target Edge Detected Image

\subsubsection{Output Image}

It is the final stage; the above segmented images (figure 9 \& figure 10) are taken as the atlases for the Multi atlas segmentation. The simple algorithm further provides the output image(figure 11) which shows the exact injury part in the brain image.

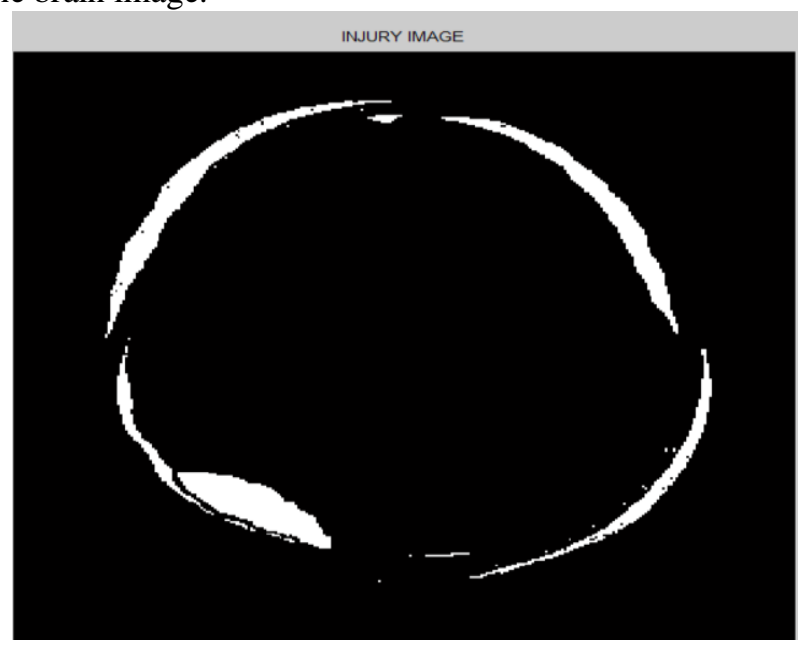

Figure 11: Output Image

\section{CONCLUSION}

Thus the tumor regions from the MR brain image are enhanced and segmented using Multi atlas segmentation method. In this proposed work, preprocessing is done with the help of histogram equalization and contrast enhancement is performed by using CLAHE. This method gives better enhancement in lesion regions than uniform distribution. Then the enhanced image is segmented with the help of multi atlas segmentation, which used multiple atlases instead of single atlas in order to avoid non-systematical errors in the labels. The experimental result shows that the proposed method accomplishes high performance with respect to MSE, PSNR and DSC.

\section{REFERENCES}

1. Gong, Tianxia, et al. "Classification of CT brain images of head trauma." IAPR International Workshop on Pattern Recognition in Bioinformatics. Springer, Berlin, Heidelberg, 2007.
2. Garima yadav, Saurabh Maheshwari and Anjali Agarwal, "Contrast limited adaptive histogram equalization based enhancement for real time video system" International Conference on advances in computing, communications and Informatics(ICACCI), 2014.

3. Rajakullayappa, P. Chinna, et al. "An Improved Image Enhancement Method Using Discrete Wavelet Transform and CLAHE."

4. Giri, Donna, et al. "Automated diagnosis of coronary artery disease affected patients using LDA, PCA, ICA and discrete wavelet transform." Knowledge-Based Systems 37 (2013): 274-282.

5. Langerak, Thomas Robin, et al. "Label fusion in atlas-based segmentation using a selective and iterative method for performance level estimation (SIMPLE)." IEEE transactions on medical imaging 29.12 (2010): 2000-2008.

6. Artaechevarria, Xabier, Arrate MunozBarrutia, and Carlos Ortiz-de-Solórzano. "Combination strategies in multi-atlas image segmentation: application to brain MR data." IEEE transactions on medical imaging 28.8 (2009): 1266-1277.

7. J.-L. Chen, J.-Y. Chang, and K.-L. Shieh, "2-d discrete signal interpolation and its image resampling application using fuzzy rule-based inference," Fuzzy Sets and Systems, vol. 114, no. 2, pp. $225-238,2000$,

8. P. Aljabar, R.A. Heckemann, A. Hammers , J.V. Hajnal , D. Rueckert ,“ Multi-atlas based segmentation of brain images: Atlas selection and its effect on accuracy", NeuroImage - 726-738,2009.

9. Jarno vane, Eero aho et.al "A HighPerformance Sum of Absolute Difference Implementation for Motion Estimation", IEEE Transactions on Circuits and Systems for Video Technology 16(7):876-883 - July 2006.

10. Nadir Nourain Dawoud, Brahim Belhaouari Samir and Josefina Janier, "Fast Template Matching Method Based Optimized Sum of Absolute Difference Algorithm for Face Localization," International Journal of Computer Applications (0975 - 8887) Volume 18-No.8, March 2011. 